\title{
Torsión anexial en una gestación espontánea. A propósito de un caso *
}

\author{
María Pineda Mateo, ${ }^{1}$ (D) Marta Romero Matas, ${ }^{2}$ (D) Angela Morales Bueno, ${ }^{1}$ \\ María del Valle Aguilar Martín. ${ }^{1}$
}

\section{RESUMEN}

La torsión anexial durante la gestación es un evento poco frecuente asociado principalmente al uso de técnicas de reproducción asistida. Ocasionalmente puede suceder en gestaciones espontáneas, sin factores predisponentes. La presentación clínica resulta, a menudo, inespecifica y, sumado a que la sensibilidad de la ecografía doppler (prueba gold estándar), es limitada, ocasiona dificultad en el diagnóstico preoperatorio. Por ello, en la mayoría de los casos es preciso visualizar de manera directa el ovario torcido para establecer la confirmación diagnóstica. De estos hechos resulta que la torsión anexial siga considerándose hoy en día, un dilema diagnóstico que no debe demorar la actitud terapéutica, pues el retraso por encima de las 48 horas se asocia frecuentemente con lesiones tisulares irreversibles. En la actualidad, la laparoscopia se impone como principal vía de abordaje quirúrgico ante la sospecha de torsión anexial en una gestante, por la seguridad y ventajas que ofrece este procedimiento.

Palabras clave: Torsión anexial, embarazo, quiste de ovario, laparoscopia ginecológica.

\section{Adnexal torsion in spontaneous pregnancy. A case report SUMMARY}

\begin{abstract}
Adnexal torsion during pregnancy is a rare event mainly associated with the use of assisted reproductive techniques. However, it can occasionally also occur in spontaneous pregnancies without any predisposing factors. Its nonspecific clinical presentation in addition to the sometimes limited sensitivity of the gold standard test, Doppler ultrasound, leads to difficulty in preoperative diagnosis. For this reason, in most cases it is necessary to perform a laparoscopy in order to directly visualize the twisted ovary to obtain diagnostic confirmation. This is why, nowadays, adnexal torsion continues to be considered a diagnostic dilemma that should not delay the therapeutic approach, since a delay over 48 hours associates several irreversible lesions of the adnexa. At present, laparoscopy is the main surgical approach in pregnant women with suspicion of adnexal torsion due to the safety and advantages it provides.
\end{abstract}

Keywords: Adnexal torsion, pregnancy, ovarian cyst, gynecological laparoscopy.

\section{INTRODUCCIÓN}

La torsión anexial es el resultado del cese del aporte sanguíneo como consecuencia de una rotación parcial o total del ovario sobre sus soportes ligamentarios. El embarazo constituye uno de los factores de riesgo de torsión anexial, fundamentalmente por la presencia del

\footnotetext{
${ }^{1}$ Unidad de Gestión Clínica de Ginecología y Obstetricia del Hospital Universitario Virgen Macarena de Sevilla, España. ${ }^{2}$ Centro Hospitalario de Alta Resolución de Lebrija. Lebrija. Sevilla, España. Correo para correspondencia: marta.6317@ gmail.com

* El trabajo ha sido presentado en la 36 edición del Congreso Nacional de la Sociedad Española de Ginecología y Obstetricia (SEGO) de 2021 en formato Póster.

Forma de citar este artículo: Pineda M, Romero M, Morales A, Aguilar M. Torsión anexial en una gestación espontánea. A propósito de un caso. Rev Obstet Ginecol Venez. 2022; 82(1): 129-135. https://doi $\operatorname{org} / 10.51288 / 00820116$
}

cuerpo lúteo, cuando su diámetro supera los $5 \mathrm{~cm}(1$ - 3) y en gestaciones conseguidas mediante técnicas de reproducción asistida (TRA) (1). No obstante, la torsión anexial durante la gestación es un cuadro muy infrecuente, con una incidencia aproximada de 1 por cada 5000 embarazos (4). El riesgo de desarrollar una torsión anexial es significativamente mayor en el primer trimestre e inicios del segundo, disminuyendo a medida que la gestación avanza (3).

El dolor abdominopélvico, en ocasiones asociado a vómitos y náuseas, constituye una forma de presentación clínica común, aunque muy inespecífica $(1,2)$. Esta situación supone un reto diagnóstico, que obliga a descartar otras condiciones causantes de esta clínica, como apendicitis, cólico renal o colecistitis (1). 
Cuando se establece la sospecha diagnóstica por la sintomatología y ante una imagen ecográfica sugerente de torsión anexial, está indicada la realización de una ecografía doppler $(1,5,6)$. La ultrasonografía doppler color constituye la prueba de imagen de primera elección para evaluar la ausencia de flujo arterial y venoso, con una especificidad destacada pero con escasa sensibilidad. Esta condición, asociada a una presentación clínica inespecífica y su baja incidencia, podría suponer un retraso diagnóstico que conlleve lesiones irreversibles en el ovario (6 - 8). Por este motivo, aun en ausencia de una confirmación diagnóstica, el abordaje quirúrgico precoz, cuando exista alta sospecha clínica, resulta fundamental para prevenir complicaciones y preservar el embarazo (8). Siempre que sea posible, es preferible la cirugía laparoscópica, cuyas indicaciones y beneficios son equivalentes a las de las pacientes no gestantes (3). En comparación con la laparotomía, los procedimientos laparoscópicos se asocian con hospitalizaciones más breves, una menor tasa de morbilidad febril, menor consumo de narcóticos y mayor comodidad para la paciente con una recuperación posoperatoria más breve $(6$, 8). Con el avance de las técnicas laparoscópicas, la seguridad y eficacia de este procedimiento queda establecido en la gestación y, ante la ausencia de contraindicaciones, constituye la vía quirúrgica de elección incluso en úteros grávidos aumentados de tamaño (9-14).

A continuación se presenta el caso de una gestación espontánea de primer trimestre complicada con torsión anexial. El objetivo del presente caso clínico es exponer el manejo diagnóstico y terapéutico con relación a la evidencia científica actual. Dado lo infrecuente de esta patología, se ha realizado una revisión de la literatura disponible en la base de datos PubMed, ${ }^{\circledR}$ entre 2006 y 2021.

\section{CASO CLÍNICO}

Se presenta el caso de una mujer de 27 años, caucásica y $\sin$ antecedentes medicoquirúrgicos familiares ni personales conocidos de interés. En cuanto a sus antecedentes ginecoobstétricos, se trataba de una paciente primigesta, mediante concepción espontánea y amenorrea de 7 semanas y 2 días. Acudió al servicio de urgencias ginecoobstétricas por dolor abdominal intenso, de inicio súbito y de escasas horas de evolución, sin otra sintomatología acompañante.

La exploración abdominal no indicó signos de alarma inmediatos como peritonismo, presentando únicamente dolor a la palpación profunda en fosa iliaca derecha. En cuanto a la especuloscopia, fue normal, sin objetivarse sangrado vía vaginal.

En la ecografía transvaginal, se evidenció una gestación intrauterina evolutiva, con un embrión con longitud craneocaudal concordante a $7+3$ semanas de amenorrea y actividad cardiaca positiva. El anexo izquierdo presentaba una ecoestructura dentro de la normalidad y en el anexo derecho se observaba una imagen de 95 x 58 milímetros (mm) que impresionó como cuerpo lúteo gestacional. Se observó una mínima cantidad de líquido libre en el fondo de saco de Douglas, con una columna vertical máxima de 20 $\mathrm{mm}$.

Se ingresó a la paciente en observación para completar el estudio y valorar la evolución clínica. Analíticamente, destacó una leucocitosis de 17 800/ $\mu \mathrm{L}$ con una neutrofilia del 86,7 \%. Las enzimas hepáticas, la proteína $\mathrm{C}$ reactiva y el sedimento de orina fueron normales.

La paciente continuó presentando dolor intenso refractario a la administración de analgesia intravenosa. 
Por este motivo, ante la sospecha de torsión anexial derecha, se amplió el estudio con ecografía doppler abdominal. El informe radiológico concluyó que no era posible descartar dicha entidad por mala visualización de los anexos y de su vascularización, dado el tamaño del útero grávido (Figura 1).

Ante estos hallazgos, se decidió realizar una laparoscopia exploradora urgente, tras explicar la indicación y el procedimiento a la paciente, así como sus beneficios y riesgos en su caso en concreto. Durante la cirugía se constató la existencia de una formación anexial de 10 centímetros $(\mathrm{cm})$ de diámetro máximo, en el ovario derecho, causante de la torsión. El ovario presentaba un aspecto totalmente necrótico con dos giros sobre sí mismo, lo que impresionaba tratarse de un cuadro subagudo en el que se había presentado un empeoramiento clínico. Ante la privación de vascularización en el anexo, tras resolver dicha torsión, se decidió realizar una ooforectomía derecha ante la imposibilidad de preservación ovárica. Durante la cirugía no se observó ninguna complicación. Ambas

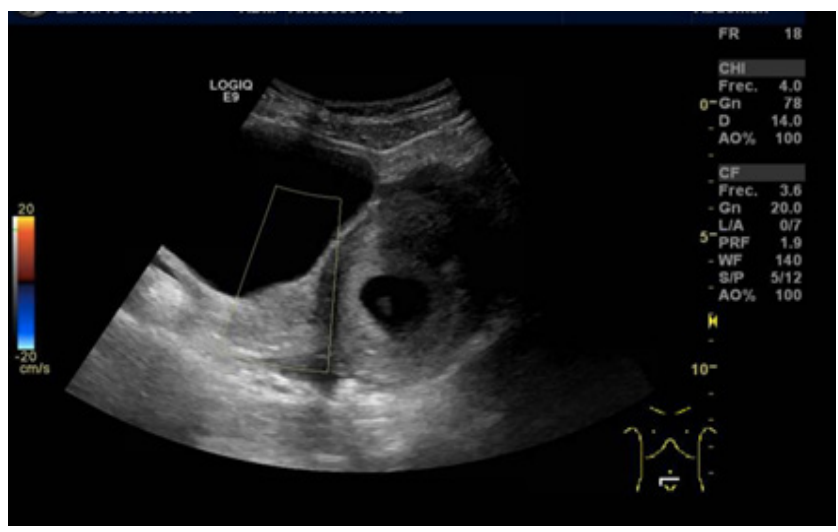

Figura 1. Ecografía Doppler pélvica con tumoración quística de $10 \mathrm{~cm}$ compatible con quiste lúteo. Hacia la derecha, útero con saco gestacional y embrión en su interior. Debido al gran tamaño del quiste, resulta difícil observar los ovarios y no se logra identificar vascularización arterial adyacente al mismo. trompas, el útero y el resto de la cavidad abdominal se veían dentro de la normalidad (Figura 2). Los hallazgos anatomopatológicos revelaron marcados cambios hemorrágicos con ectasia vascular atribuible a la torsión ovárica.

La paciente tuvo un posoperatorio sin incidencias y tras el alta hospitalaria la gestación evolucionó favorablemente, constatándose mediante ecografía. Inmediatamente tras la cirugía, se inició tratamiento con progesterona de 400 microgramos $(\mu \mathrm{g})$ cada 12 horas vía vaginal para suplementar la función del cuerpo lúteo gestacional hasta la finalización del primer trimestre de gestación. El seguimiento del embarazo se intensificó en el posoperatorio, con una visita programada a los 8 días de la intervención y tras comprobar ausencia de complicaciones, se indicó control habitual de la gestación con próxima visita de control gestacional a las 12 semanas. Sin embargo, la paciente fue valorada previamente en urgencias en dos

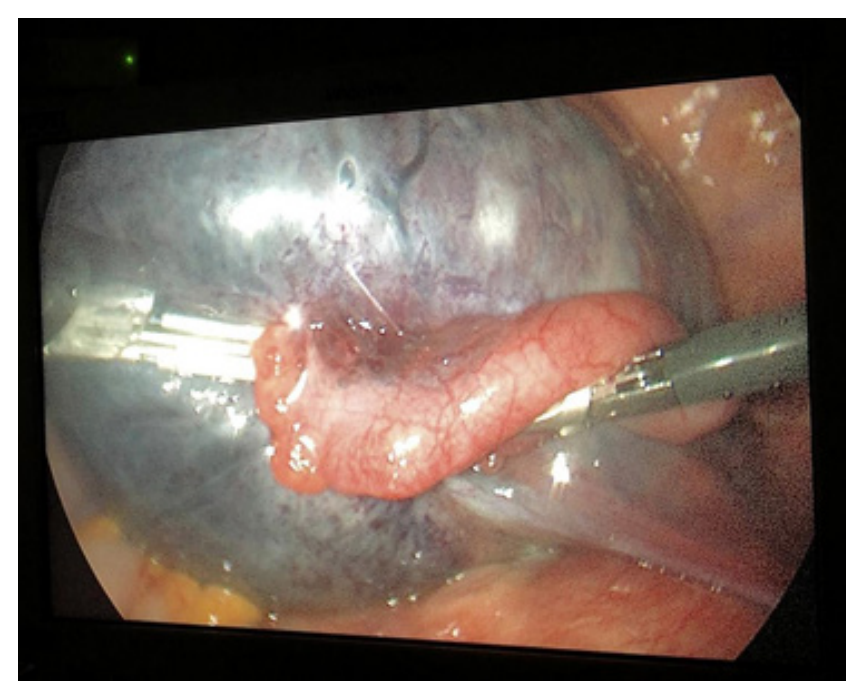

Figura 2. Imagen laparoscópica en la que se observa un quiste ovárico de aspecto benigno y gran tamaño $(10 \mathrm{~cm})$ que ha provocado la torsión anexial derecha y provocado su necrosis. Trompa derecha ligeramente edematosa, que logra preservarse. 
ocasiones por presentar episodios de sangrado genital en el primer trimestre, autolimitado espontáneamente, y con relación a la presencia de un hematoma retrocorial de $28 \times 10 \mathrm{~mm}$, visualizado por ecografía, y que se resolvió sin incidencias. La primera consulta protocolizada de embarazo se realizó a las $12+2$ semanas de amenorrea, se comprobó gestación viable, con actividad cardiaca presente, longitud craneocaudal concordante a 13 semanas y persistencia de hematoma retrocorial con el mismo diámetro que presentaba al diagnóstico. En esta visita se indicó suspender tratamiento con progesterona por el desplazamiento lúteo-placentario e iniciar controles protocolizados del embarazo. A partir del segundo trimestre, el embarazo tuvo una evolución común. Se realizaron controles gestacionales habituales a las 20 y 32 semanas y precisó seguimiento a las 36 semanas para valorar el crecimiento fetal por sospecha de macrosomía. Tras descartarse por el peso fetal estimado en la biometría, la paciente fue derivada a consulta de alto riesgo obstétrico a las 39+3 semanas para valoración de inducción del parto si presentara una gestación en vía de prolongación.

Finalmente la paciente ingresó por urgencias obstétricas en la semana $40+3$ de amenorrea por inicio de trabajo de parto de forma espontánea, finalizando en parto instrumentado mediante ventosa por una segunda fase del parto prolongada. El recién nacido fue a término sin incidencias, con buena puntuación en el test de apgar (10 al primer, quinto y décimo minuto de vida) y peso adecuado para edad gestacional (3600 g).

\section{DISCUSIÓN}

La torsión anexial constituye la quinta emergencia quirúrgica de causa ginecológica más común (4). Según algunos estudios, del $10 \%$ al $20 \%$ ocurre en embarazadas $(4,7)$. Con respecto a la población no gestante, se estima un riesgo dos veces mayor, que va disminuyendo cuanto mayor es la edad gestacional (2, 4). El incremento del estímulo hormonal presente en la edad fértil de la mujer y en el periodo concepcional es responsable de la mayor incidencia descrita en dichas etapas (3).

Según los datos revisados, y concordante con el caso clínico reportado, la torsión anexial es más frecuente en el lado derecho, lo cual está probablemente relacionado con la limitación de la movilidad que ofrece el colon sigmoide en el lado izquierdo $(1,2)$.

Resulta un hecho que los factores de riesgo tradicionales para la torsión anexial están correlacionados con un mayor volumen de los anexos. Hasta en el $70 \%$ de los casos, las masas anexiales identificadas al principio de la gestación son quistes funcionales que van desapareciendo (2). Por ello, la indicación quirúrgica de resecabilidad se ha establecido ante masas sintomáticas, sospechosas de malignidad o cuando su tamaño supera los $10 \mathrm{~cm}$ de manera persistente (2). Así pues, el riesgo de torsión anexial se vio incrementado en el caso de esta gestante, dado el tamaño alcanzado por el cuerpo lúteo gestacional.

Otra importante causa, actualmente en auge, es el síndrome de hiperestimulación ovárica presente hasta en el $16 \%$ de las gestaciones conseguidas mediante TRA (4). Hoy en día, el uso de inductores de la ovulación en tratamientos de fertilidad es cada vez más frecuente $\mathrm{y}$, a consecuencia de ello, se observa una tendencia ascendente del riesgo de torsión anexial en el embarazo $(5-7,9)$.

Es reseñable que, a pesar de que el embarazo sea considerado un potencial factor de riesgo, es un evento poco frecuente y actualmente de incidencia incierta debido a la escasez de estudios publicados. Especialmente se encuentra gran interés en considerar el caso clínico expuesto, en el que la gestación se consiguió de manera espontánea sin exposición al 
uso de gonadotropinas como tratamiento inductor de la ovulación, tal y como ocurre en la mayoría de los casos reportados $(6,7)$.

El diagnóstico temprano y el manejo quirúrgico adecuado de la torsión anexial es la única forma de prevenir complicaciones y preservar la función futura de los ovarios (12). Sin embargo, el diagnóstico preoperatorio de la torsión anexial es dificultoso, especialmente durante la gestación. La forma de presentación típica es la aparición de dolor pélvico, de inicio súbito en la mayoría de los casos, que en ocasiones, como en la paciente que se presenta, puede tener intervalos de mejoría en el contexto de una torsión subaguda. Este dolor se asocia a menudo con náuseas, vómitos y fiebre. Otro hallazgo relativamente frecuente es la palpación de una masa a nivel pélvico $(4,5)$. Ahora bien, esta sintomatología resulta inespecífica. Por un lado, hay que considerar la presentación de manera fisiológica en la gestación; y por otro, obliga a realizar un diagnóstico diferencial ante patología urgente que cursa con un cuadro de abdomen agudo en el embarazo, tal y como es la apendicitis, la colecistitis o un cólico renal (13). Se ha demostrado la asociación entre la duración de los síntomas, el grado de torsión y la necrosis. Por lo tanto, se requiere un alto índice de sospecha para realizar el diagnóstico precoz y evitar un retraso por encima de las 36-48 horas, en cuyo caso se hace más improbable revertir el daño tisular $(1,3$, 4).

Ante esta sintomatología inespecífica, el uso de pruebas complementarias podría orientar al diagnóstico, sin embargo se ha evidenciado que solo en un $46 \%$ de los casos la ecografía facilita su detección preoperatoria (3). La prueba de imagen de elección es la ecografía doppler abdominopélvica. La ausencia de flujo arterial y venoso demostrada por ecografía, es el signo más característico para considerar la torsión ovárica como primera posibilidad diagnóstica. Sin embargo, a pesar de su alta especificidad, su sensibilidad es menor, entre el $46 \%$ y el $75 \%$ (5), pues hay que tener en consideración que el ovario presenta un flujo sanguíneo dual y por lo tanto la presencia de flujo sanguíneo normal en la ecografía no excluye el diagnóstico de torsión anexial. Se estima que hasta en el $60 \%$ de los casos de torsión ovárica comprobadas quirúrgicamente tenían flujo sanguíneo evidenciado por ecografía (6). Además, como en este caso clínico, el útero grávido puede comprometer una correcta observación de los anexos y que el resultado de la ecografía no arroje información concluyente. En estas ocasiones en las que la ecografía resulta indeterminada, se ha propuesto la resonancia magnética (RMN) como prueba complementaria adicional. La RMN presenta la ventaja de ofrecer un mayor campo de visión, mejor contraste, mayor precisión y mayor capacidad para distinguir masas anexiales. Sin embargo, sus notables limitaciones como el coste y el tiempo necesario para su realización hacen que se desaconseje su uso de manera rutinaria y en situación de emergencia $(2,8)$.

Por ello, se puede concluir que el diagnóstico definitivo de torsión anexial se realiza mediante visualización directa del anexo torcido (1). Los hallazgos clínicos y/o ecográficos facilitan la decisión de llevar a cabo una evaluación quirúrgica. La intervención no debe retrasarse por encima de las 48 horas ya que a mayor tiempo de evolución es mayor la probabilidad de encontrar lesiones irreversibles, y por ello la cirugía conservadora se vuelve improbable $(1,3)$. Por este motivo, destaca la importancia de tener en consideración esta patología para evitar el retraso diagnóstico (1). Por tanto, el objetivo de la cirugía es confirmar la sospecha clínica y evaluar la viabilidad del anexo afectado y preservarlo, si es posible. En el caso de esta paciente, aunque refería dolor abdominal los días previos, no consultó en urgencias hasta que este fue más intenso y asociado a vómitos y malestar, lo cual ocasionó un retraso diagnóstico. Dado el empeoramiento clínico y la alta sospecha de torsión anexial se estableció la indicación quirúrgica sin poder preservar el ovario, por las repercusiones de un cuadro con una evolución posible a lo largo de varios días. 
La vía de abordaje quirúrgico en la gestación ha sido un tema controvertido desde hace años. Sin embargo, en la actualidad se ha demostrado la eficacia y seguridad de la laparoscopia para el manejo de la torsión anexial, sin ocasionar daños en la madre ni en el feto (10). En comparación con la laparotomía, las ventajas de la laparoscopia en la gestación no difieren de las de las mujeres no gestantes. Ocasiona menos dolor posoperatorio, lo que implica menor necesidad de analgésicos, menor probabilidad de íleo posoperatorio, estancias hospitalarias más cortas, con una alimentación y deambulación más precoz (10). Todas estas ventajas se pudieron observar en la paciente que se presenta, cuya estancia hospitalaria en el posoperatorio fue de apenas 48 horas y cuya recuperación se produjo rápidamente y sin incidencias destacables. Con relación a la gestación, la laparoscopia ofrece dos ventajas específicas: por un lado disminuye la manipulación uterina durante el procedimiento, y por otro lado se evita que la cicatriz de la laparotomía vaya sufriendo los cambios provocados por el aumento del útero grávido, de modo que disminuye las molestias maternas y ocasiona menor tasa de hernias incisionales $(10,11,13)$. Si bien las indicaciones no difieren de la población no gestante, sí que existen recomendaciones específicas a la hora de realizar el procedimiento quirúrgico laparoscópico, adoptando una serie de modificaciones en las embarazadas. El objetivo es proceder de la manera menos invasiva posible, consiguiendo una adecuada visualización del campo quirúrgico sin perturbar el curso del embarazo $(13,14)$. Estas modificaciones incluyen variar la postura de la paciente durante la cirugía con un ligero posicionamiento en decúbito lateral izquierdo, minimizando la compresión de la arteria aorta y la vena cava. Además, se recomienda usar técnicas de entrada abierta o colocación de trócares bajo visión directa, evitando aquellos trócares con puntas muy punzantes. También se debe adaptar la inserción de los puertos a la altura del fondo uterino, evitando la entrada a nivel umbilical, y mantener una presión intraabdominal entre 8 y $12 \mathrm{~mm}$ de $\mathrm{Hg}$, con el fin de evitar alterar el flujo sanguíneo útero-placentario. Igualmente, también es importante intentar reducir al máximo el tiempo operatorio y la presión de dióxido de carbono con la intención de prevenir una acidosis materno-fetal (12 - 14).

Otra particularidad a reseñar en el caso clínico expuesto es la extirpación del cuerpo lúteo gestacional ante la imposibilidad de preservación ovárica. El quiste lúteo es el responsable de la producción de progesterona durante las primeras 10 semanas de embarazo. Por este motivo, las recomendaciones actuales residen en evitar la extirpación del mismo. Sin embargo, dado que en ocasiones no resulta posible, se considera necesaria la suplementación con progesterona con el objetivo fundamental de preservar el embarazo. La administración puede realizarse en comprimidos vaginales o menos frecuentemente, como inyección intramuscular. La dosis recomendada es aproximadamente de $100 \mu \mathrm{g}$ cada 8 o 12 horas, con posibilidad de incrementarla hasta $800 \mu \mathrm{g}$ diarios, sin que exista evidencia disponible en la actualidad sobre el beneficio que aporta dicho incremento. La suplementación se debe considerar hasta las 10 semanas de la gestación, momento en el que la placenta suple la función del cuerpo lúteo (5). En este caso se decidió prolongar el tratamiento hasta la semana 12 por la presencia de un hematoma retrocorial sintomático. Tras su suspensión, el resto de la gestación transcurrió sin incidencias destacables, con un resultado obstétrico favorable.

\section{CONCLUSIONES}

A pesar de la baja incidencia de torsión anexial en la gestación, resulta fundamental tenerla en consideración como posibilidad diagnóstica en una gestante con dolor abdominal, independientemente de las semanas de amenorrea y de que existan o no factores predisponentes. Los cambios fisiológicos y el diagnóstico diferencial con otras enfermedades 
que cursan con dolor abdominal no deben demorar la actitud terapéutica, para poder mejorar los resultados de preservación anexial. En la actualidad, la laparoscopia constituye la principal vía de abordaje quirúrgico por su eficacia y su seguridad en la gestación.

\section{Sin conflictos de interés.}

\section{REFERENCIAS}

1. Hasiakos D, Papakonstantinou K, Kontoravdis A, Gogas L, Aravantinos L, Vitoratos N. Adnexal torsion during pregnancy: report of four cases and review of the literature. J Obstet Gynaecol Res. 2008; 34(4Pt2):683687. doi: 10.1111/j.1447-0756.2008.00907.x.

2. Kumari I, Kaur S, Mohan H, Huria A. Adnexal masses in pregnancy: a 5-year review. Aust N Z J Obstet Gynaecol. 2006; 46(1):52-54. doi: 10.1111/j.1479828X.2006.00515.x.

3. Seo SK, Lee JB, Lee I, Yun J, Yun BH, Jung YS, Chon SJ. Clinical and pathological comparisons of adnexal torsion between pregnant and non-pregnant women. J Obstet Gynaecol Res. 2019; 45(9):1899-1905. doi: 10.1111/jog. 14057.

4. Yuk JS, Shin JY, Park WI, Kim DW, Shin JW, Lee JH. Association between pregnancy and adnexal torsion: A population-based, matched case-control study. Medicine (Baltimore). 2016; 95(24):e3861. doi: 10.1097/MD.0000000000003861.

5. Runowicz CD, Brewer M. Adnexal mass in pregnancy [Internet]. Massachusetts: Uptodate Whaltam; 2019 [consultado septiembre de 2020]. Disponible en: https://www.uptodate.com/contents/adnexal-mass-inpregnancy?search $=$ Adnexal + mass + in + pregnancy $+\& s$ ource $=$ search $\_$result\&selectedTitle $=1 \% 7 \mathrm{E} 150 \&$ usage type $=$ default\&display_rank $=1$

6. Sun Y, Feng G, Fu Y, You J, Li M, Zhu Y. Emergent complication of assisted reproductive technology: Clinical analysis of 17 pregnant women with adnexal torsion. Am J Emerg Med. 2020; 38(2):305-308. doi: 10.1016/j.ajem.2019.158391.
7. Rackow BW, Patrizio P. Successful pregnancy complicated by early and late adnexal torsion after in vitro fertilization. Fertil Steril. 2007; 87(3):697.e9-12. doi: 10.1016/j.fertnstert.2006.05.091.

8. Bai W, Xu X, Xie H, Sun C, Che K, Liu M, et al. Adnexal torsion in the third trimester of pregnancy: a case report and diagnostic value of MR imaging. BMC Med Imaging. 2020; 20(1):19. doi: 10.1186/s12880020-00422-1.

9. Tsai HC, Kuo TN, Chung MT, Lin MY, Kang CY, Tsai YC. Acute abdomen in early pregnancy due to ovarian torsion following successful in vitro fertilization treatment. Taiwan J Obstet Gynecol. 2015; 54(4):438441. doi: 10.1016/j.tjog.2013.08.013.

10. Kahramanoglu I, Eroglu V, Turan H, Kaval G, Sal V, Tokgozoglu N. Isolated adnexal torsion in a 20 -week spontaneous twin pregnancy. Int J Surg Case Rep. 2016; 23:138-140. doi: 10.1016/j.ijscr.2016.04.029.

11. Aydin T, Yucel B. Laparoscopic management of adnexal torsion in a twin, in vitro fertilization pregnancy at 23 weeks. Wideochir Inne Tech Maloinwazyjne. 2014; 9(4):655-657. doi: 10.5114/wiitm.2014.45732. .

12. Chang SD, Yen CF, Lo LM, Lee CL, Liang CC. Surgical intervention for maternal ovarian torsion in pregnancy. Taiwan J Obstet Gynecol. 2011; 50(4):458-462. doi: 10.1016/j.tjog.2011.10.010.

13. Troncoso JL, Ricci P, Susaeta R, Devoto JC. Torsión anexial y embarazo: resolución laparoscópica mínimamente invasiva, con entrada bajo visión directa. Rev Chil Obstet Ginecol. 2008; 73(4):277-282. http:// dx.doi.org/10.4067/S0717-75262008000400011.

14. Stany MP, Elkas JC. Laparoscopic surgery in pregnancy [Internet]. Massachusetts: Uptodate Whaltam; 2019 [consultado septiembre de 2020]. Disponible en: https://www.uptodate.com/contents/ search?search=Laparoscopic $\% 20$ surgery $\% 20$ in $\% 20$ pregnancy. 